\title{
Shape control of active surfaces inspired by the movement of euglenids
}

\author{
Marino Arroyo \\ LaCàN, Universitat Politècnica de Catalunya-BarcelonaTech \\ Barcelona 08034, Spain
}

Antonio DeSimone

SISSA, 34136 Trieste, Italy

June 6, 2013

\begin{abstract}
We examine a novel mechanism for active surface morphing inspired by the cell body deformations of euglenids. Actuation is accomplished through in-plane simple shear along prescribed slip lines decorating the surface. Under general non-uniform actuation, such local deformation produces Gaussian curvature, and therefore leads to shape changes. Geometrically, a deformation that realizes the prescribed local shear is an isometric embedding. We explore the possibilities and limitations of this bioinspired shape morphing mechanism, by first characterizing isometric embeddings under axisymmetry, understanding the limits of embeddability, and studying in detail the accessibility of surfaces of zero and constant curvature. Modeling mechanically the active surface as a non-Euclidean plate (NEP), we further examine the mechanism beyond the geometric singularities arising from embeddability, where mechanics and buckling play a decisive role. We also propose a non-axisymmetric actuation strategy to accomplish large amplitude bending and twisting motions of elongated cylindrical surfaces. Besides helping understand how euglenids delicately control their shape, our results may provide the background to engineer soft machines.
\end{abstract}

\section{Introduction}

Soft machines are structures made out of flexible active materials that undergo controllable shape transformations under the action of a variety of stimuli. Examples include swellable hydrogels sensitive to either temperature (Hu et al., 1995) or electric-field (Kwon et al., 2008), electroactive polymers (Jager et al., 2000), liquid-crystal elastomers (Warner and Terentjev, 2007; DeSimone and Teresi, 2009) actuated by temperature (deHaan et al., 2012), light 
(White et al., 2008; Camacho-Lopez et al., 2004), or electric field (Fukunaga et al., 2008), and biohybrid constructs consisting of muscle cells attached to elastomer sheets (Feinberg et al., 2007; Nawroth et al., 2012). Bioinspiration plays an important role in this field.

Often, actuation is accomplished through bending, e.g. by non-uniform swelling through the thickness, typically in quasi one-dimensional structures (Hu et al., 1995; Armon et al., 2011; Sawa et al., 2010; Urayama, 2012). In quasi two-dimensional structures, imposed bending alone, even if two-dimensional, provides access to a limited repertoire of shapes as it can only produce curvature in one direction due to the dominant stretching penalty of doubly curved shapes (Warner et al., 2010). An alternative actuation paradigm for thin films consists of imposing an in-plane non-uniform deformation field. If such an imposed deformation cannot be realized within the plane, it leads to shape transformations (Sharon

At the root of this concept, coined as non-Euclidean plates (NEPs), is Gauss' Egregium theorem, by which a generic in-plane deformation (metric tensor) produces Gaussian curvature. Geometrically, a target nonuniform metric is prescribed on the surface representing the thin film. Such metric can sometimes be realized by an isometric embedding, i.e. a parametrized surface in space with the specified metric. In this situation, the thin body will change shape to meet the target metric. Although in-plane elasticity overwhelmingly dominates bending elastic is the main shape select play a role in some multiple isometric e through bending eneror
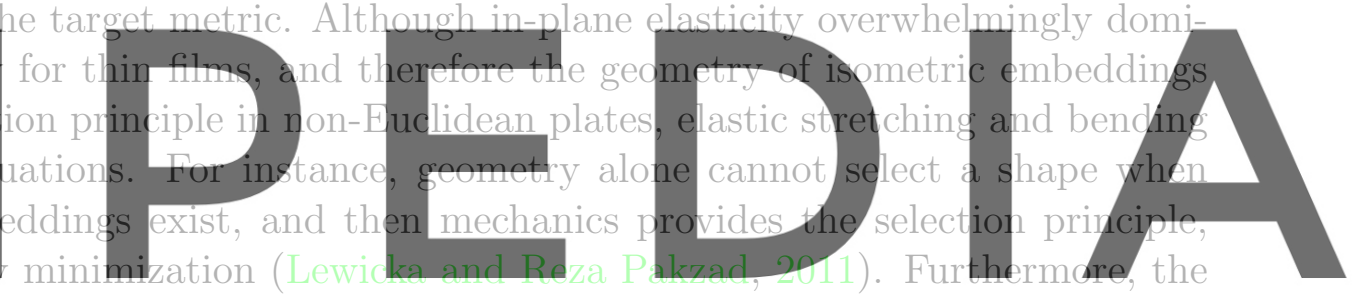

target metric may not be realizable, leading to frustrated shapes that may relax in-plane

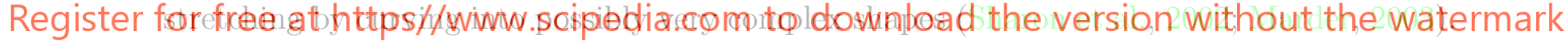

This viewpoint has been motivated by the shapes of flowers and leaves as a result of non-uniform growth (

et al., 2004; Liang and Mahadevan, 2009; Amar et al., 2012), and has been implemented in disk and tubular topologies by non-uniform swelling/shrinkage of gels (Klein et al., 2007; Kim et al., 2012) or non-uniform switching of liquid crystal elastomers (deHaan et al., 2012; Sawa et al., 2010; Urayama, 2012). In these and most references, the non-uniformity in the deformation is accomplished by patterning non-uniform swelling or nematic director fields, see also e.g. Modes et al. (2011), and applying a uniform stimulus, although it has also been accomplished by non-uniform illumination (Camacho-Lopez et al., 2004). Such programmed NEPs can therefore execute predefined shape changes, although the path between configurations can present significant variability (Kim et al., 2012).

Here, inspired by the motility of euglenids, we examine another mechanism for actuating the target metric, which we have recently identified (Arroyo et al., 2012). Euglenids are a family of unicellular protists present worldwide in a wide range of aquatic environments, with typical sizes from tens to hundreds of microns (Leander, 2008; Triemer, 1999). They are very diverse in terms of morphology, flexibility, feeding strategy (feeding on other cells, photosynthesis, absorbing dissolved nutrients directly from the environment), or motility, and 


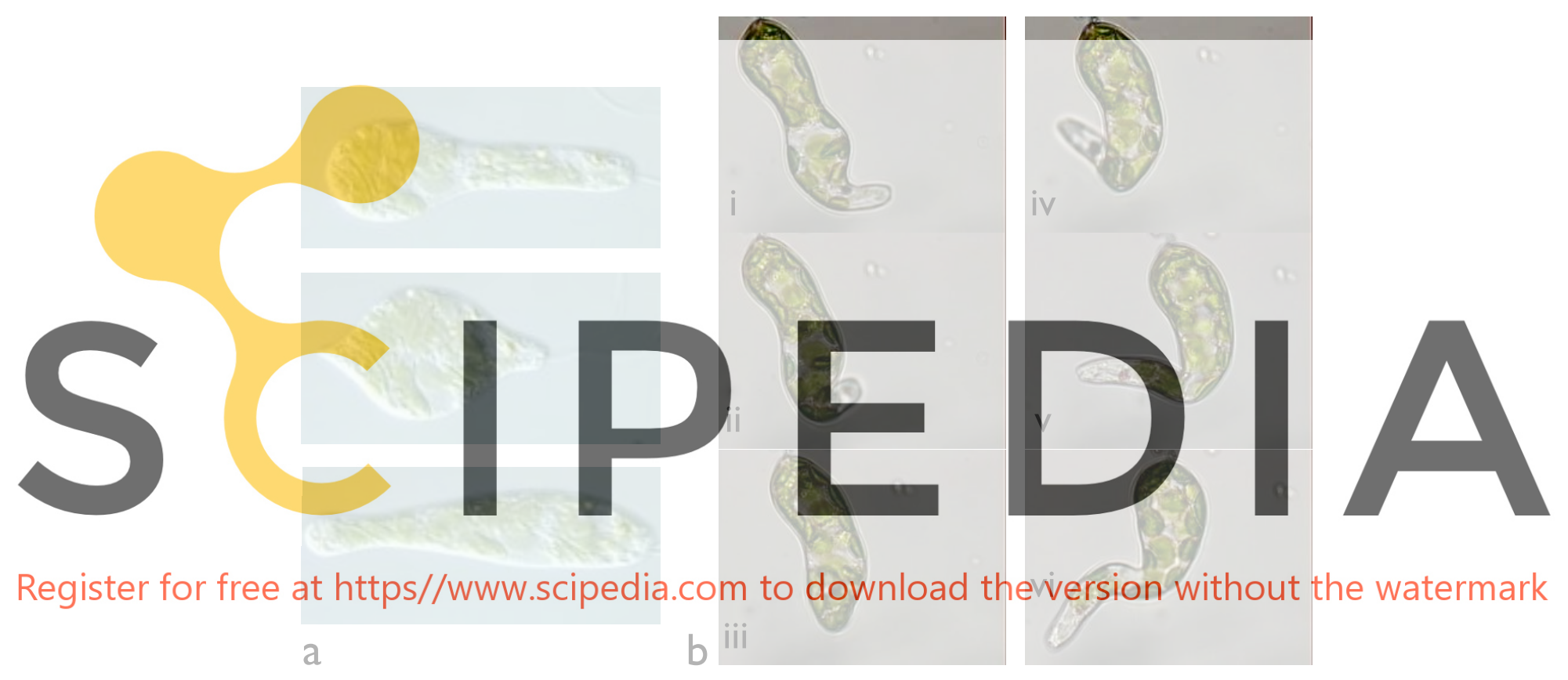

Figure 1: Motility of plastic euglenids. (a) Eutreptiella species executing highly repetitive and nearly axisymmetric stroke. Original frame images courtesy of Richard E. Triemer. (b) Euglena species performing a more vacillating motion, including bending and twisting of the cell. Original frame images courtesy of Francisco Pujante. 
can be solitary or live in colonies. Although euglenids move primarily beating their flagella, some species exhibit large amplitude and highly concerted body distortions, called metaboly or euglenoid movement, which have fascinated microbiologists and physical scientist from the earliest days of microscopy to now (Dobell, 1932; Fletcher and Theriot, 2004). See Figure 1 for an illustration. The function of metaboly remains unclear, although it seems to have emerged from the need of a malleable cell wall to engulf large prey, and may have found utility in locomotion later in the evolutionary history. For this reason, metaboly would have persisted in photosynthetic and osmotrophic species, which do not engulf other cells. We have previously shown that metaboly is a slow but efficient motility mode in a Newtonian fluid, and suggested that it seems particularly well-suited for locomotion in complex or granular media (A rroyo et al., 2012). Metaboly is controlled by the pellicle, a striated envelope enclosing most euglenids, see Figure 2.

The cell shape excursions of metaboly are gracefully executed in time-scales of a few seconds, and include peristaltic motions, bending, twisting, rounding, and elongating (see Figure 1 for an illustration). The euglenoid movement is very smooth in space and time, suggesting that it does not involve geometrical instabilities. The striking fact that a unicellular organism, with minimal anatomical and sensory machinery, is able to perform in a seemingly controlled manner such diverse motions with remarkable agility suggests that metaboly is grounde we developed a contin analyze movie record revisit and expand this theory to systematic this shape actuation mechanism, focusing on a

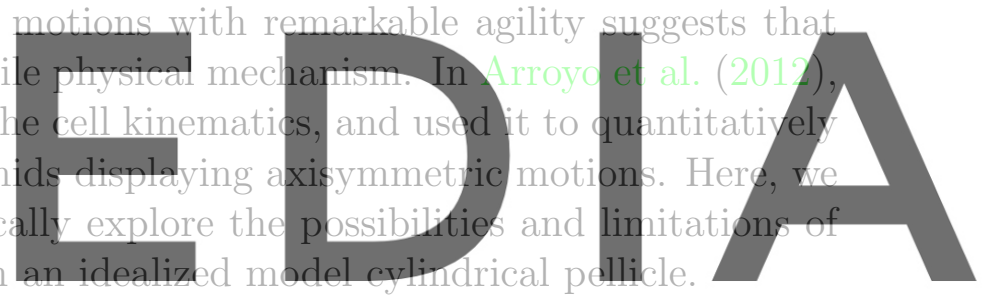
Register for frefat https//.www.scipedia.com to download the version without the watermark

The pellicle is an active striated surface that executes metaboly, see Figure 2 a,b. There is strong anatomical and functional evidence suggesting that this active envelope can reconfigure by mobilizing molecular motors along microtubulues arranged below the pellicle strips, see Figure 2 c. More specifically, the pellicle strips have been shown to slide relative to each other during metaboly without changing their width or length (Suzaki and Williamson, 1985, 1986). Since the flexible pellicles of deformable euglenids exhibit a large number of strips (a few tens), we adopt a continuum approximation and idealize the kinematics as consisting of simple shear along the pellicle strips. For highly localized deformation features, in the length scale of the width of the pellicle strips, such a model may miss effects arising from the discrete nature of the pellicle. We develop next a mathematical model for the pellicle kinematics.

Consider a material surface $\Gamma_{0} \subset \mathbb{R}^{3}$, parametrized as $\boldsymbol{x}_{0}(u, v)$ for $(u, v) \in \bar{\Gamma}$, modeling the pellicle at a given reference state. The natural basis of the tangent bundle of the reference surface is obtained by partial differentiation (denoted with a comma) with respect to $u$ and $v$, $\left\{\boldsymbol{x}_{0, I}, I=u, v\right\}$. By $\boldsymbol{e}_{i}, i=1,2,3$ we denote the Cartesian basis vectors. We consider now a deformed configuration $\boldsymbol{x}(u, v)=\sum_{i=1}^{3} x^{i}(u, v) \boldsymbol{e}_{i}$, parametrizing the surface $\Gamma$. The in-plane 

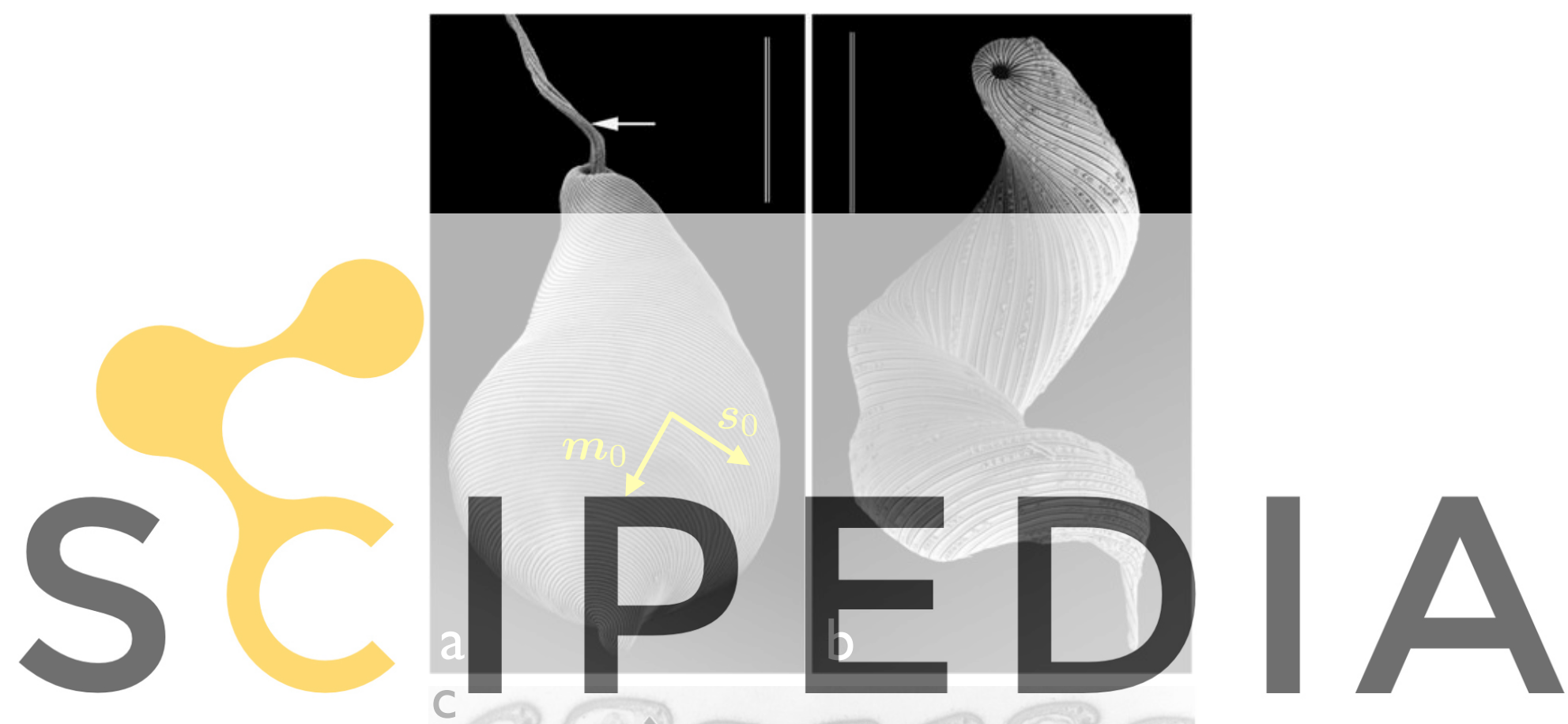

Register for free at https//www.scipedia.com to download the version without the watermark

Figure 2: Scanning electron micrographs of Eutreptia pertyi (a) and Euglena spirogyra (b) (Scalebar $=10 \mu \mathrm{m}$ ), showing the pellicle, a striated envelope covering most euglenids. Amongst euglenids, flexible species exhibit a large number of strips (tens), whose helicity correlates with body deformation. (c) Transmission electron micrograph of the substructural features of the euglenid pellicle (here Peranema trichophorum, scalebar $=1 \mu \mathrm{m}$ ). The pellicle is a cortical complex including the plasma membrane, a set of interlocking proteinaceous strips, microtubules, and tubular cisternae of endoplasmic reticulum arranged along the strips. Micrographs from Leander et al. (2001). We also illustrate the tangent vector fields $\boldsymbol{s}_{0}$ and $\boldsymbol{m}_{0}$ along and perpendicular to the pellicle strips. 
deformation gradient is a $2 \times 2$ tensor field mapping the the tangent bundle of $\Gamma_{0}$ to that of $\Gamma$, and can be expressed as $\boldsymbol{F}(P)=D \boldsymbol{x}\left(\boldsymbol{x}_{0}^{-1}(P)\right)\left[D \boldsymbol{x}_{0}\left(\boldsymbol{x}_{0}^{-1}(P)\right)\right]^{-1}$ for $P \in \Gamma_{0}$ (Marsden and Hughes, 1983). By expressing $D \boldsymbol{x}_{0}$ and $D \boldsymbol{x}$ in the canonical basis of $\bar{\Gamma}$ and the natural bases of $\Gamma_{0}$ and $\Gamma$, their components are the identity matrix, and therefore the components of the right Cauchy-Green deformation tensor in the basis $\left\{\boldsymbol{x}_{0, I}, I=u, v\right\}$ are equal to those of the surface metric tensor, and given by the scalar products of tangent vectors to the deformed surface

$$
C_{I J}=g_{I J}=\boldsymbol{x}_{, I} \cdot \boldsymbol{x}_{, J} .
$$

The surface $\Gamma_{0}$ is decorated by a tangential unit vector field $\boldsymbol{s}_{0}$, whose integral lines can be thought of as the continuum pellicle strips. With the continuum approximation to the pellicle, we suppose that the active surface is capable of producing simple shear $\gamma(u, v)$ along $\boldsymbol{s}_{0}$. If we denote by $\boldsymbol{m}_{0}$ a unit vector field perpendicular to $\boldsymbol{s}_{0}$, the in-plane deformation gradient due to the pellicle shear can be written as

$$
\boldsymbol{F}=\boldsymbol{R}\left(\boldsymbol{I} \boldsymbol{d}+\gamma \boldsymbol{s}_{0} \otimes \boldsymbol{m}_{0}\right),
$$

where $\mathbb{R}$ is an undetermined rotation tensor field, which can be avoided by considering the

right Cauchy-Green deformation tensor
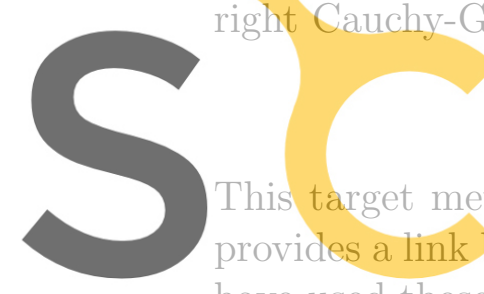

This target metric has unit
provides a link between actua

the spatio-temporal pat
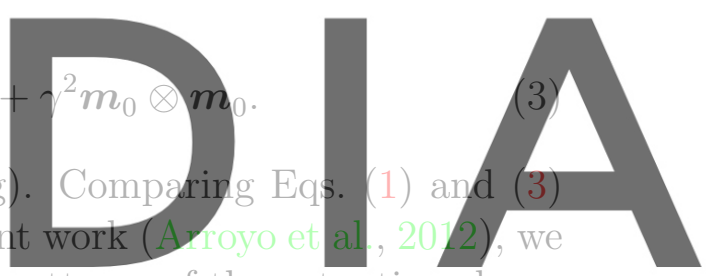

Register for $(u, t)$. from video recordinas of. motile cells. Fioure 3 illustrates the correlation between Register for free at https/Ww. Wcipedia.com to download the version without the watermark shape changes and pellicle shear actuation for the peristaltic motion of a motile Euglenid exhibiting an axisymmetric motion captured in video and processed with the method presented in this reference. Here, we examine the shapes resulting from a prescribed pellicle shear field, which is tantamount to finding a global isometric embedding in $\mathbb{R}^{3}(\boldsymbol{x})$ of a Riemannian 2-manifold with the prescribed metric tensor given by Eq. (3).

It is worth comparing this shape actuation mechanism with another area-preserving mechanism given by the right stretch tensor $\boldsymbol{U}=1 / \lambda \boldsymbol{q}_{0} \otimes \boldsymbol{q}_{0}+\lambda \boldsymbol{p}_{0} \otimes \boldsymbol{p}_{0}$, where $\boldsymbol{p}_{0}$ and $\boldsymbol{q}_{0}$ are mutually orthogonal vector fields. This mechanism can be relevant for thin films made of nematic glasses or elastomers (Modes et al., 2011). By choosing $\boldsymbol{p}_{0}$ and $\boldsymbol{q}_{0}$ so that they form a $45^{\circ}$ angle with $\boldsymbol{s}_{0}$ and $\boldsymbol{m}_{0}$, it is easy to see that $\boldsymbol{U}^{2}$ is only equivalent to Eq. (3) around infinitesimal deformations, while for finite deformations the two mechanisms are fundamentally different.

For concreteness, we fix throughout the paper a cylindrical reference configuration of length $L_{0}$, radius $R_{0}$, and whose pellicle strips are straight and aligned with its axis, see Figure 4-i for an illustration. This minimal model system is representative of euglenids, and potentially interesting in applications. We can conveniently choose $u$ (aligned with the axis of the cylinder) and $v$ (along the azimuthal direction) such that $\boldsymbol{x}_{0, u}=\boldsymbol{s}_{0}$ and $\boldsymbol{x}_{0, v}=\boldsymbol{m}_{0}$. Then, given a function $\gamma(u, v)$, a deformed configurations that realizes the prescribed simple 


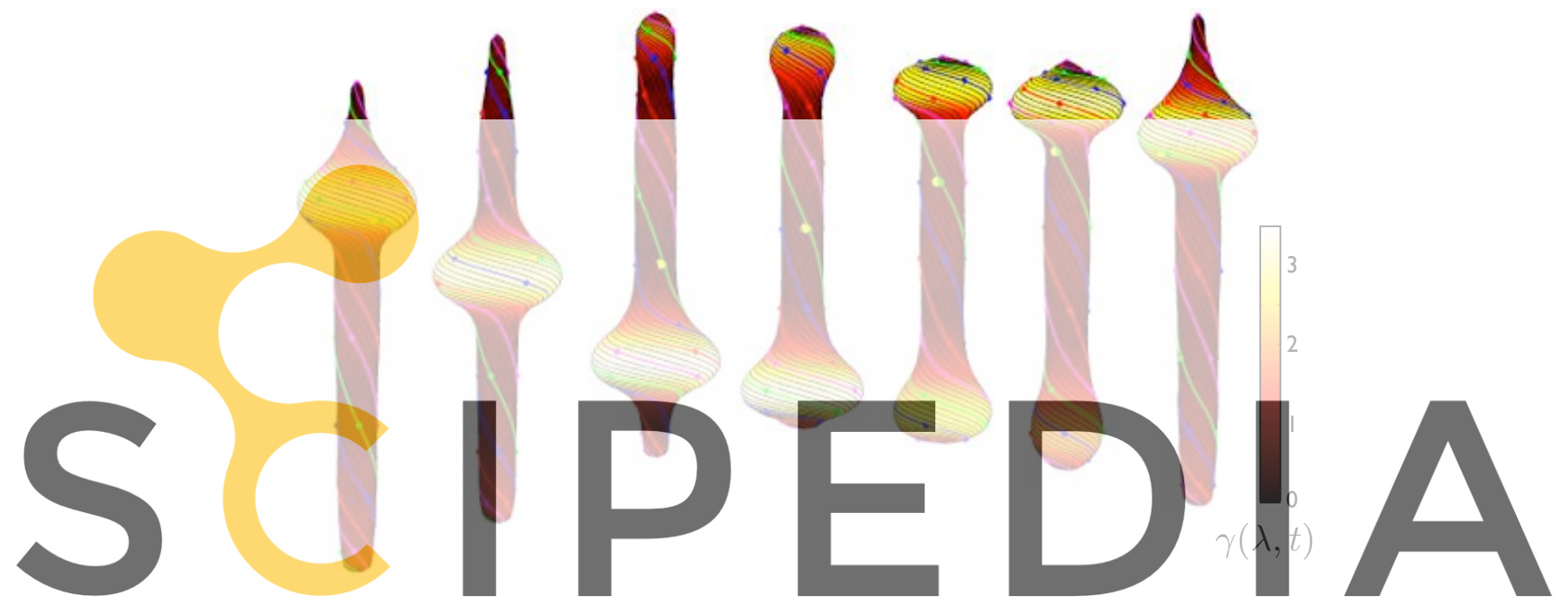

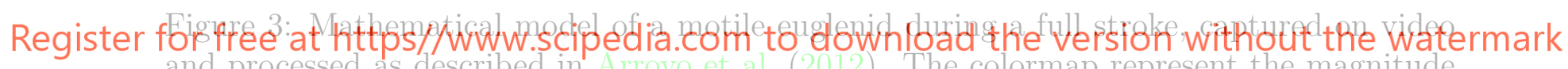
and processed as described in Arroyo et al. (2012). The colormap represent the magnitude

of the pellicle shear, which reaches over 300\%, the black lines are pellicle strips, and the colored lines and points are Lagrangian markers, which help visualize the net rotation of the cell around its symmetry axis. During the power stroke of metaboly, a bulge slides down the elongated cell, accomplishing significant forward motion when placed on a Newtonian fluid at vanishing Reynolds number. During the recovery stroke, the bulge at the tail disappears at the expense of the bulge at the head, involving strong cytoplasmic streaming, and the cell moves back a little. The figures highlights the strong correlation between shape, pellicle helicity, and azimuthal motions. 
shear along the pellicle should satisfy the three independent nonlinear partial differential equations in

$$
\left[\begin{array}{cc}
\boldsymbol{x}_{, u} \cdot \boldsymbol{x}_{, u} & \boldsymbol{x}_{, u} \cdot \boldsymbol{x}_{, v} \\
\boldsymbol{x}_{, u} \cdot \boldsymbol{x}_{, v} & \boldsymbol{x}_{, v} \cdot \boldsymbol{x}_{, v}
\end{array}\right]=\left[\begin{array}{cc}
1 & \gamma \\
\gamma & 1+\gamma^{2}
\end{array}\right] .
$$

The target metric in the right-hand side of this equation is not flat in general. Its Gaussian curvature (the product of the principal curvatures) can be computed by differentiating the components of the metric tensor as (do Carmo, 1976)

$$
K=\left(\gamma_{, v}-\gamma \gamma_{, u}\right)_{, u}
$$

The system in Eq. (4) is highly nontrivial and many open questions remain (Han and Hong, 2006). The equations change character depending on the sign of the Gaussian curvature associated with the target metric. Existence of global solutions cannot be expected in general, and when they exist they can be non unique. A trivial example is $\gamma=0$, realizable by infinitely many cylindrical surfaces with non-circular cross-section. To analyze local and global existence of isometric embeddings, Eqs. (4) have been recast in equivalent forms, as a Monge-Ampère equation called the Darboux equation, or as the Mainardi-Codazzi system. Here, we are interested in the global problem for non-compact surfaces, and for Gaussian curvatures changing stead, we proceed by the differential equat examining non-axisy of non-Euclidean plates
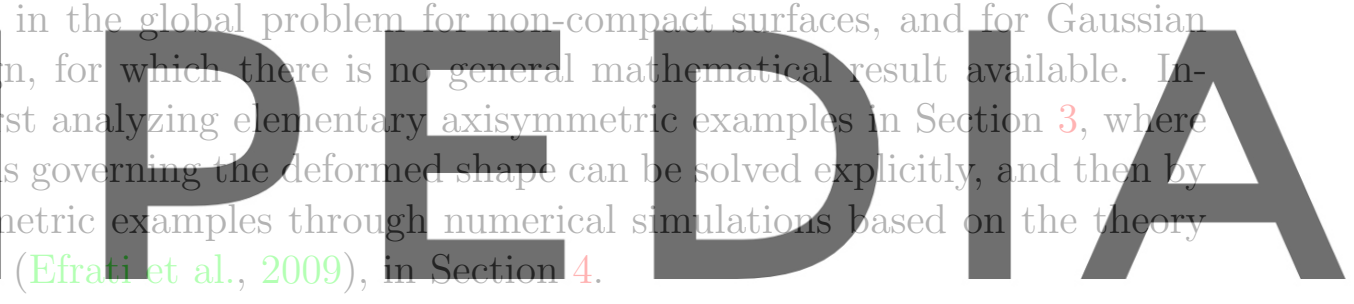

\section{Register for free at https//www.scipedia.com to.download the version without the watermark}

We examine here simple shapes resulting from axisymmetric actuation, i.e. $\gamma_{, v}=0$, in which the target metric can be met exactly. Thus, mechanically, such deformations involve no stretch, which is the dominant energetic contribution for thin bodies. The most trivial example, uniform pellicle shear, helps understand how simple shear along the pellicle strips results in shape changes. Figure 4 provides a pictorial depiction of this situation, which exploits the fact that $K=0$, and therefore the pellicle surface can be developed onto a plane. From elementary geometrical considerations, the final cylinder has length $L_{0} / \sqrt{1+\gamma^{2}}$ and radius $R_{0} \sqrt{1+\gamma^{2}}$. This example also illustrates the strong correlation between radius and local pellicle orientation observed in micrographs of euglenids, see Figures 2 and 3, as well as the coupling between shape changes and azimuthal motions.

\subsection{Kinematics}

We parametrize the reference configuration as

$$
\boldsymbol{x}_{0}(u, v)=\left\{R_{0} \cos \left(v / R_{0}\right), R_{0} \sin \left(v / R_{0}\right), u\right\}, \quad u \in\left[0, L_{0}\right], \quad v \in\left[0,2 \pi R_{0}\right]
$$




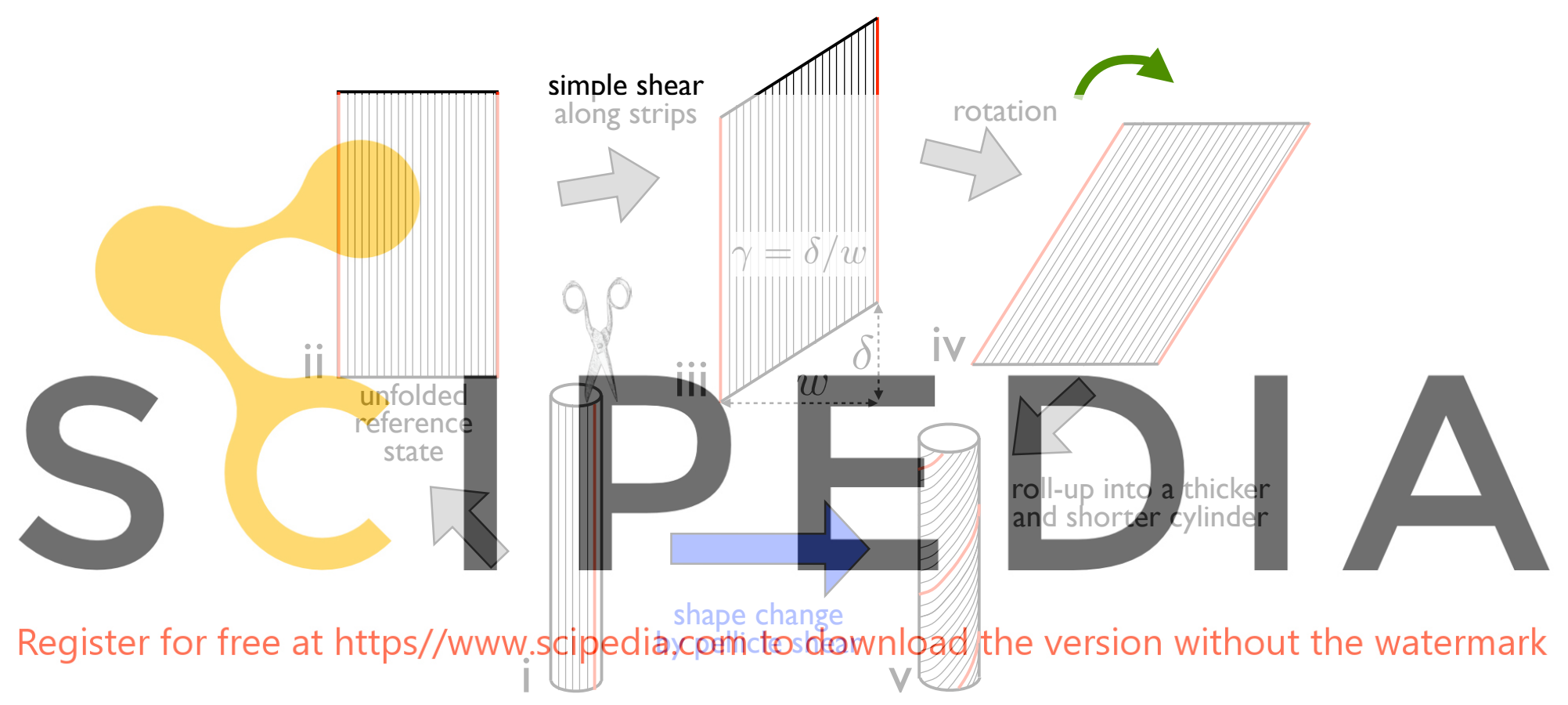

Figure 4: Main idea of the shape actuation principle by simple shear along the pellicle strips decorating the active surface. In the simplest situation, the reference shape is a cylinder with the pellicle lines oriented parallel to its axis, and the prescribed shear is uniform. Uniform pellicle shear results in a shorter and thicker cylinder with helical pellicle strips. This can be understood by cutting and unrolling the reference pellicle (i) into a planar rectangle (ii), shearing it uniformly (iii), and then rolling the resulting parallelogram along a direction perpendicular to the free ends (iv) to produce the deformed shape (v). 
so that $\boldsymbol{x}_{0, u}=\boldsymbol{s}_{0}$ and $\boldsymbol{x}_{0, v}=\boldsymbol{m}_{0}$ are unit vectors. To model axisymmetric shapes, the deformed configuration can be written in terms of its generating curve in the symmetry plane, $\{r(u), z(u)\}$, and the azimuthal displacement $\psi(u)$ as

$$
\boldsymbol{x}(u, v)=\left\{r(u) \cos \left[v / R_{0}+\psi(u)\right], \quad r(u) \sin \left[v / R_{0}+\psi(u)\right], \quad z(u)\right\} .
$$

Then, Eqs. (4) become

$$
\left[\begin{array}{cc}
r^{\prime 2}+z^{\prime 2}+r^{2} \psi^{\prime 2} & r^{2} \psi^{\prime} / R_{0} \\
r^{2} \psi^{\prime} / R_{0} & \left(r / R_{0}\right)^{2}
\end{array}\right]=\left[\begin{array}{cc}
1 & \gamma \\
\gamma & 1+\gamma^{2}
\end{array}\right]
$$

where $\gamma(u)$ and $(\cdot)^{\prime}$ denotes differentiation with respect to $u$. It then follows that

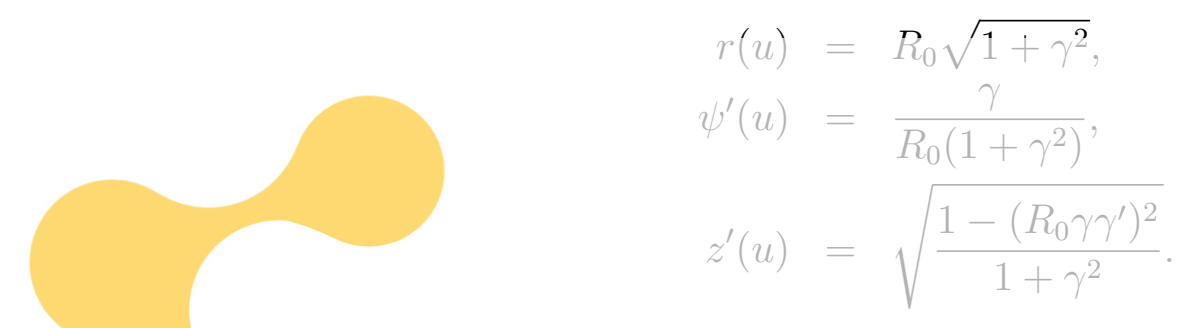

These equations can be easily integrated, analytically or numerically, to yield an isometric embedding. It immediad der the radical sign in of the pellicle shear

When equality holds, $\boldsymbol{s}^{\prime}(u)$
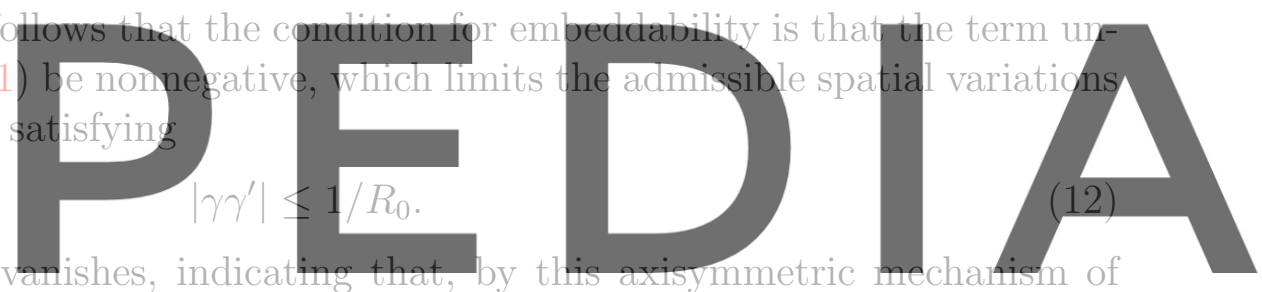

surface deformation, it is not possible to fold back the surface, i.e. $r(z)$ should remain single

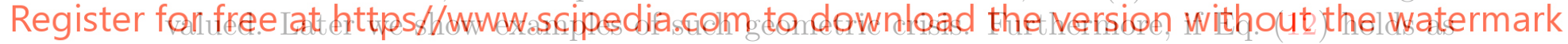

a strict inequality, the isometric embedding is unique up to rigid body motion, e.g. the integration constants in Eqs. (10,11). Regarding the accessibility of shapes, it is worth noting that Eq. (9) implies that the local radius is always greater than or equal to $R_{0}$, the radius when the pellicle strips lack helicity.

Finally, we recall standard relations in differential geometry of surfaces (do Carmo, 1976) to compute the curvature of the deformed surface. From Eq. (7), the coefficients of the first fundamental form are $E=r^{\prime 2}+z^{\prime 2}, F=0$ and $G=\left(r / R_{0}\right)^{2}$, and those of the second fundamental form are $e=\left(r^{\prime} z^{\prime \prime}-r^{\prime \prime} z^{\prime}\right) / \sqrt{r^{\prime 2}+z^{\prime 2}}, f=0$ and $g=z^{\prime} r /\left(R_{0}^{2} \sqrt{r^{\prime 2}+z^{\prime 2}}\right)$. The Gaussian curvature is $K=\left(e g-f^{2}\right) /\left(E G-F^{2}\right)$, from which we recover Eq. (5), and the mean curvature is $H=(e G+g E) /(2 E G)$, from which we compute the principal curvatures $k_{1}=e / E$ and $k_{2}=g / G$, along meridians and parallels respectively.

\subsection{Cones and flat annular regions}

A natural question is to seek for nontrivial shapes of zero Gaussian curvature. By setting $K=0$ in Eq. (5) in the axisymmetric context, we find $\gamma \gamma^{\prime}=c s t$, from which we obtain

$$
\gamma(u)=\sqrt{A(1-\xi)+B \xi},
$$




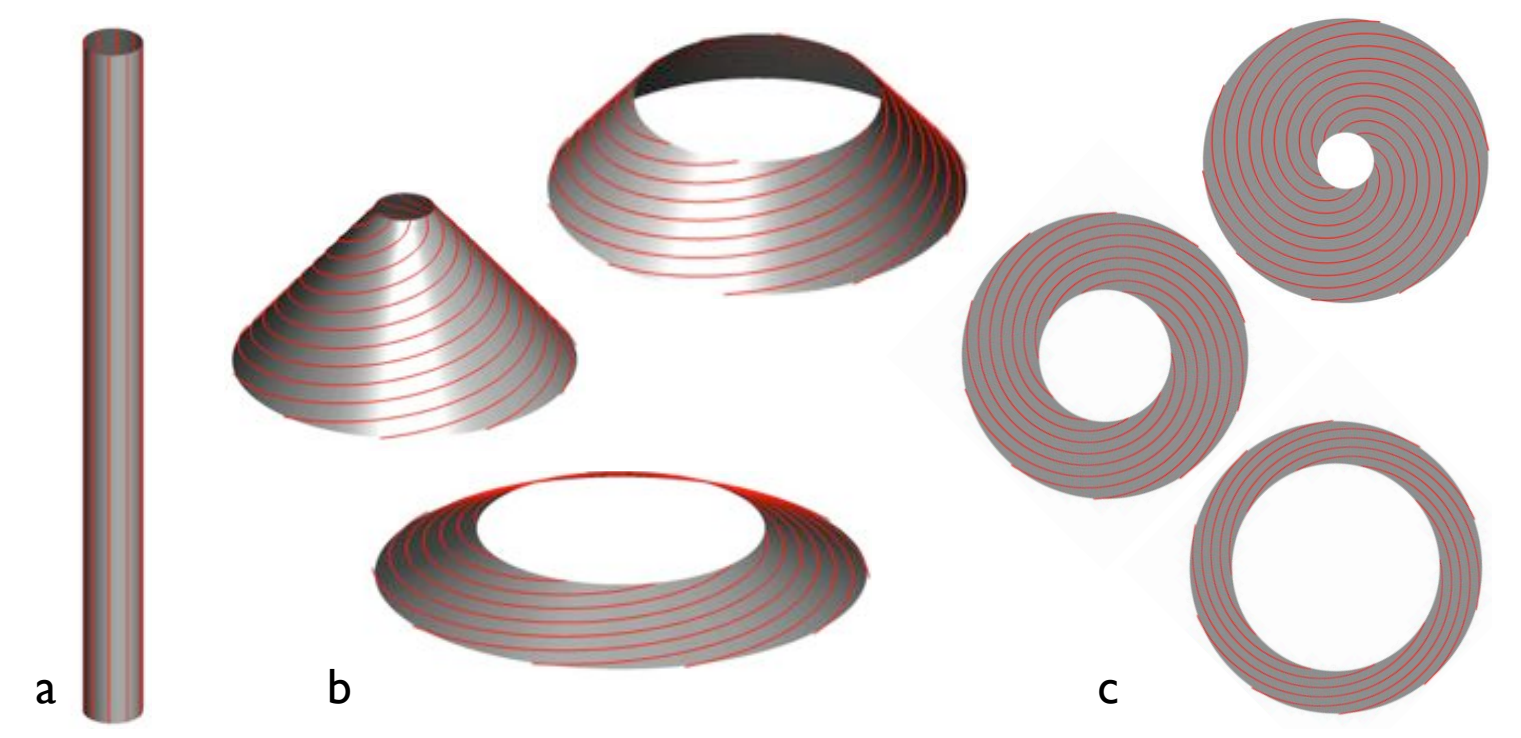

Figure 5: Cylindrical pellicle (a) actuated by shear distributions with square root dependence along the axis, resulting in truncated cones (b), or flat annular regions (c). In (c), the annuli have been scaled differently. Otherwise, all other plots are in the same scale. Note that all these surfaces have the same area.

where for convenience we rescale $u$ such that $0 \leq \xi \leq 1$ and $\xi^{\prime}(u)=1 / L_{0}$. The nondimensional constants $A$ and $B$ should be non-negative, and embeddability restricts the choices of parameters as $|B-A| \leq 2 L_{0} / R_{0}$, which follows from Eq. (12). The case $A=B$ results in cylinders, as illustrated in Figure 4 . From Eqs. $(9,11)$, it is easy to check that

$$
\frac{z^{\prime}(u)}{r^{\prime}(u)}=\frac{1}{B-A} \sqrt{\left(\frac{2 L_{0}}{R_{0}}\right)^{2}-(B-A)^{2}}
$$

is independent of $u$, and consequently the resulting shapes are truncated cones. Recalling Eq. (9), it is clear that the parameters $A$ and $B$ are related the radii of the rims of the surface as $r(0)=R_{0} \sqrt{1+A}$ and $r\left(L_{0}\right)=R_{0} \sqrt{1+B}$, which allows us to rewrite the embeddability condition as

$$
\left|r^{2}(0)-r^{2}\left(L_{0}\right)\right| \leq 2 L_{0} R_{0} .
$$

When equality holds, as clear from Eq. (14), the resulting surface is a flat annular region. Figure 5 illustrates a few examples, with a depiction of the deformed pellicle strips.

\subsection{Spheres and pseudospheres}

We seek now embeddings with constant Gaussian curvature $K=\bar{K} / L_{0}^{2}$, where we introduce for convenience the non-dimensional Gaussian curvature $\bar{K}$. From Eq. (5), we find that this is the case for

$$
\gamma(u)= \pm \sqrt{-\bar{K} \xi^{2}+A \xi+B},
$$


where $A$ and $B$ are non-dimensional constants.

\section{Spherical surfaces $(\bar{K}>0)$}

For concreteness, we examine symmetric surfaces with respect to $z$, leading to $A=\bar{K}$. All other surfaces are truncated versions of this family of surfaces. Then, the term under the radical sign in Eq. (16) is non-negative for all $\xi \in[0,1]$ if $B \geq 0$. Since now $\gamma \gamma^{\prime}=$ $\bar{K} / L_{0}(1 / 2-\xi)$, the embeddability condition in Eq. (12) is satisfied everywhere in the domain when $\bar{K} \leq 2 L_{0} / R_{0}$. When $\bar{K}$ is equal to the critical value, a singularity occurs at the ends of the tube.

Spherical surfaces can be classified as spindles, spheres or bulges depending on whether the principal curvature in the azimuthal direction is larger, equal, or smaller than the principal curvature along the meridian. Since $K$ is the product of principal curvatures, the sign of $S=k_{2}^{2}-K$ characterizes spindles $(S>0)$, spheres $(S=0)$ and bulges $(S<0)$, where the principal curvature along parallels can be computed as

$$
k_{2}=\frac{z^{\prime}}{r \sqrt{r^{\prime 2}+z^{\prime 2}}}
$$

which follows from the results at the end of Section 3.1. Recalling Eq. (16), $A=\bar{K}$ and $B \geq 0$, we find that the sign of $S$ is that of

$$
\left(\frac{L_{0}}{R_{0}}\right)^{2}-\bar{K}\left(1+B+\frac{\bar{K}}{4}\right)
$$

which classifies the two-parameter family of spherical surfaces given by $\bar{K}$ and $B$.

\section{Pseudospheres $(\bar{K}<0)$}

Focusing for concreteness on surfaces with zero pellicle shear at one end (other surfaces are truncated versions or unions of those examined here), we can write the pellicle shear as

$$
\gamma(u)= \pm \sqrt{\xi(A-\bar{K} \xi)}
$$

Enforcing (1) that the expression under the radical sign remains nonnegative and (2) that the embeddability condition in Eq. (12) holds for all $\xi \in[0,1]$, we find the conditions

$$
0 \leq A \leq 2\left(\frac{L_{0}}{R_{0}}+\bar{K}\right)
$$

Figure 6 provides a joint phase-diagram, for both positive and negative curvature, of the surfaces of constant Gaussian curvature accessible from a reference cylindrical pellicle. Starting with surfaces of positive curvature, we have represented spindles of increasing curvature, from left to right, with $B=0$ (i.e. $r(0)=r\left(L_{0}\right)=R_{0}$ ), three spheres of different 


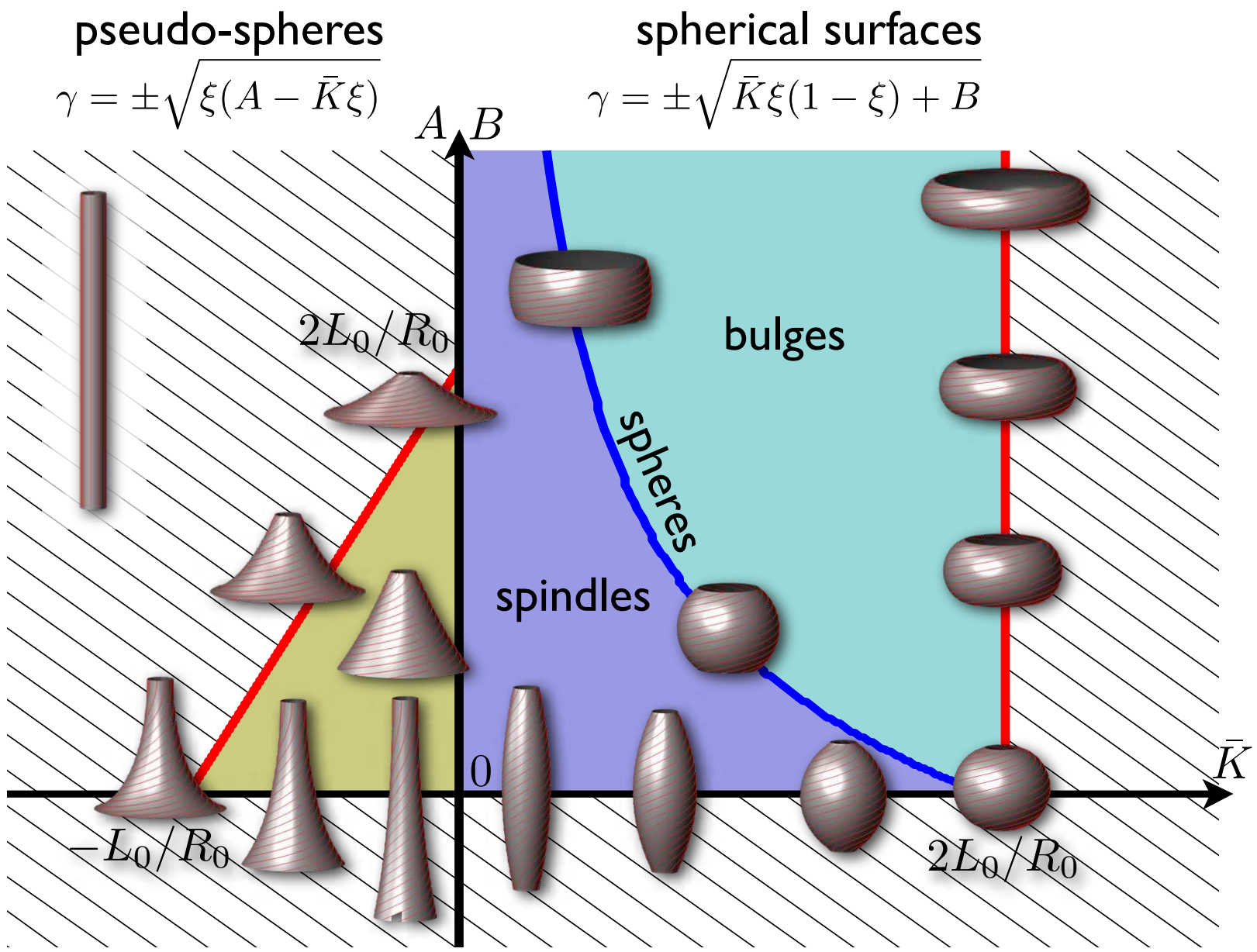

Figure 6: Phase diagram of the surfaces of constant negative (left) and positive (right) Gaussian curvature that can be achieved by imposing a pellicle shear distribution on a cylindrical pellicle (top-left). All surfaces are represented in the same scale. The dashed region denotes forbidden parameter choices. The phase regions are delimited by boundaries set by the embeddability condition in Eq. (12) (red lines), by the fact that, as shown in Eq. (9), the radius cannot be smaller than $R_{0}$ (horizontal axis), and by the surfaces of zero curvature illustrated in Figure 5 (vertical axis). The surfaces on the red boundaries exhibit rim singularities. The boundary between spindles and bulges (blue line) is given by Eq. (18), and its intersection with the horizontal axis depends on the aspect ratio of the cylinder as $\bar{K}=2\left(-1+\sqrt{1+\left(L_{0} / R_{0}\right)^{2}}\right)$. 


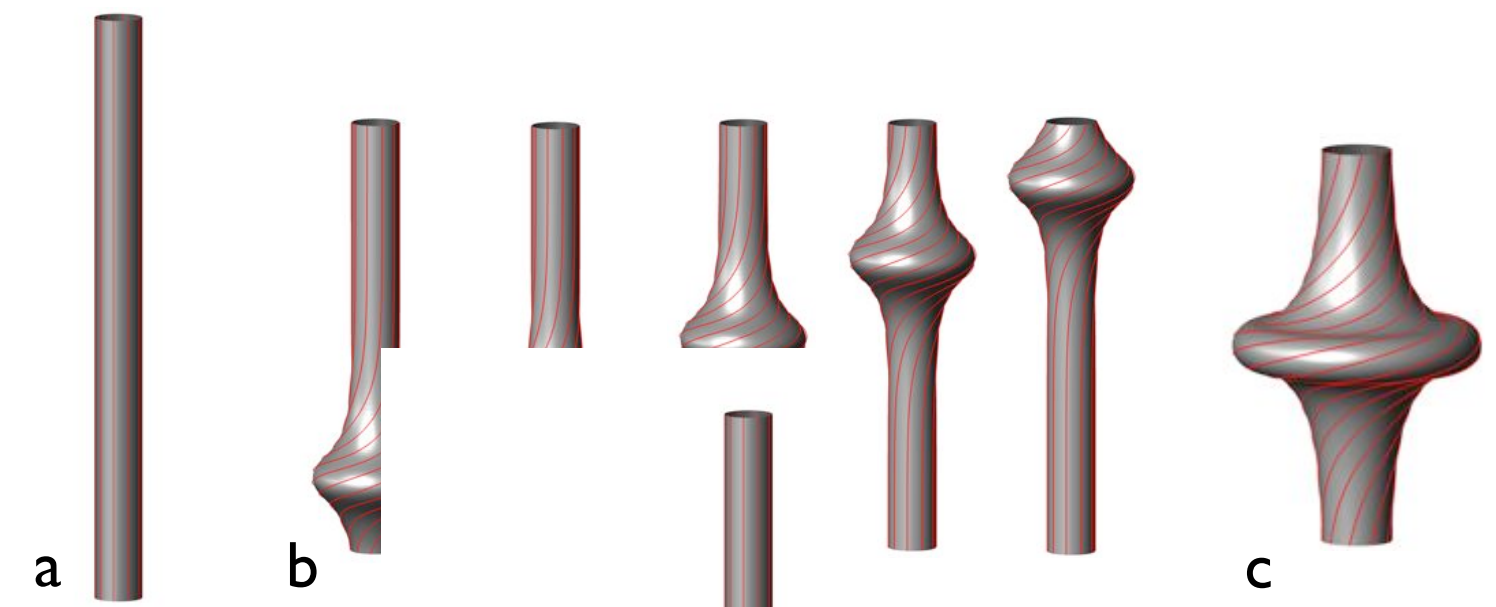

Figure 7: A pellicle shear distribution uniform along the azimuthal direction and with a Gaussian dependence along the axis of the reference surface (a) results in a localized bulge. Peristaltic motions can be achieved by moving the Gaussian actuation along the tube (b). (c) depicts a surface at the verge of the geometric singularity, with two parallels where $z^{\prime}=0$.

curvature, and bulges of maximum curvature $\left(\bar{K}=2 L_{0} / R_{0}\right)$ and different values of $B$. All the bulges are on the red boundary, and therefore exhibit a singularity $\left(z^{\prime}=0\right)$ at their rims. As for the surfaces of negative curvature, the three surfaces on the red boundary exhibit a singularity at the lower rim, while the three surfaces at the horizontal axis $(A=0$ and linear dependence of $\gamma(u)$ ) can be extended smoothly on the top by joining them to their reflexion with respect to a horizontal plane.

\subsection{Localized deformations}

To mimic the peristaltic deformation shown in Figure 3 and exhibited by some euglenid species, we consider now a localized pellicle shear with Gaussian dependence

$$
\gamma(u)=A \exp \left[-\left(\frac{\xi-C}{B}\right)^{2}\right],
$$

where the non dimensional parameters $A, B>0$, and $C$ determine the strength, width and location of the perturbation. The maxima of $\left|\gamma \gamma^{\prime}\right|$ occur for $\xi=C \pm(1 / 2) B$, and consequently the embeddability condition in Eq. (12) is met everywhere if

$$
A^{2} \leq \sqrt{e} B \frac{L_{0}}{R_{0}} .
$$

Figure 7 illustrates how the radius and helicity can be modulated locally in this way. It also shows a surface at the verge of the geometric singularity, obtained when equality holds in Eq. (22). Interestingly, such a non-uniform localized deformation can be approximated by 
juxtaposing cylindrical, spherical, and pseudo-spherical surfaces. In particular, the surface in Figure 9(c) is close to a bulge connected to two pseudospheres, all of which have rim singularities.

\section{Beyond embeddability and axisymmetry with non- Euclidean plates}

We now examine the euglenoid actuation mechanism beyond the strict embeddability constraint of the previous section, or beyond axisymmetry. For this purpose, we adopt the elastic theory of non-Euclidean plates (Efrati et al., 2009), and find the shapes resulting from minimizing the discrepancy between the actual and the target metrics, in the presence of a small bending energy. We summarize below the theory of NEPs, and implement it numerically with finite elements. We then examine the resulting shapes when a pellicle is brought beyond the singularities identified in the previous section. Finally, we explore non-axisymmetric actuation strategies, which result in bending and twisting.

\subsection{Non-Euclidean plates}

Recall that $\Gamma_{0}$ denotes the reference pellicle, and $\boldsymbol{x}(u, v)$ a deformed configuration of the pellicle surface, as described in Section 2. Adapting Efrati et al. (2009), we write the elastic energy of the NEP as

$$
E[\boldsymbol{x}]=\int_{\Gamma_{0}} w_{2 D}(\boldsymbol{C}, \mathcal{K} ; \overline{\boldsymbol{C}}) d S_{0}
$$

where $w_{2 D}$ is the strain energy density detailed later, $\boldsymbol{C}=\boldsymbol{F}^{T} \boldsymbol{F}$ is the actual right CauchyGreen deformation tensor, $\boldsymbol{C}$ is the target deformation given by Eq. (3). By $\boldsymbol{N}$ we denote a unit normal to $\Gamma$ and by $\mathcal{K}$ the pull-back of the second fundamental form $b_{I J}=\boldsymbol{x}_{, I J} \cdot \boldsymbol{N}$ to $\Gamma_{0}$, which is the curvature measure compatible with $\boldsymbol{C}$ (Arroyo and Belytschko, 2002). For instance, the mean curvature is computed as $H=\mathcal{K}: \boldsymbol{C}$. The distinction between $\mathcal{K}$ and $\boldsymbol{b}$ is important in the numerical implementation, where $\{u, v\}$ are the finite element coordinates. In this case, the natural basis vectors $\left\{\boldsymbol{x}_{0, u}, \boldsymbol{x}_{0, v}\right\}$ do not coincide with a meaningful frame, e.g. given by $\boldsymbol{s}_{0}$ and $\boldsymbol{m}_{0}$, and $D \boldsymbol{x}_{0}$ is element-dependent and different from the identity. More details are given in Arroyo and Belytschko (2004).

In the simplest model, the reduced strain energy density derived from a bulk isotropic Kirchhoff-Saint Venant constitutive model with Kirchhoff-Love's thin shell assumptions has a membrane and a bending contribution, and takes the form

$$
w_{2 D}(\boldsymbol{C}, \mathcal{K} ; \overline{\boldsymbol{C}})=w_{m}(\boldsymbol{C} ; \overline{\boldsymbol{C}})+w_{b}(\mathcal{K} ; \overline{\boldsymbol{C}})=\frac{t}{2} \mathcal{A}^{I J K L} E_{I J} E_{K L}+\frac{t^{3}}{24} \mathcal{A}^{I J K L} \mathcal{K}_{I J} \mathcal{K}_{K L}
$$

where $t$ is the shell's thickness, $\boldsymbol{E}=1 / 2(\boldsymbol{C}-\overline{\boldsymbol{C}})$,

$$
\mathcal{A}^{I J K L}=\lambda\left[\bar{C}^{-1}\right]^{I J}\left[\bar{C}^{-1}\right]^{K L}+\mu\left(\left[\bar{C}^{-1}\right]^{I K}\left[\bar{C}^{-1}\right]^{J L}+\left[\bar{C}^{-1}\right]^{I L}\left[\bar{C}^{-1}\right]^{J K}\right),
$$


and $\lambda$ and $\mu$ are Lame's constants. In this model, the curvature energy is relative to a flat state, although it could measure curvature deviations from $\Gamma_{0}$ in a theory of non-Euclidean shells.

Let us assume first that $\overline{\boldsymbol{C}}$ is embeddable. If $t$ is small, the bending energy imposes a small bias to the stretching energy, and therefore minimization of the elastic energy is expected to lead to a surface that closely realizes the target metric. By reducing $t$, we expect to converge to an exact embedding of the target metric, and therefore the scaled membrane energy $E_{m} / t=(1 / t) \int_{\Gamma_{0}} w_{m} d S_{0}$ should tend to zero. See Lewicka and Reza Pakzad (2011) for mathematical results along these lines. If the target metric is not embeddable, the mechanics of the NEP will select morphologies that approximate the target metric, but with finite scaled membrane energy even as $t$ tends to zero. In this situation, the frustrated embeddings depend fundamentally on the choice of $t$, and the theory of NEP can be regarded as a plausible mechanical model for the actual system, rather than a device to explore the geometry of isometric embeddings.

Here, we implement this theory numerically with subdivision finite elements, and minimize the elastic energy in Eq. (23) with a limited memory BFGS quasi-Newton algorithm (Arroyo and Belytschko, 2004), which results in at least locally stable equilibrium configurations. It must be noted that beyond embeddability, we may expect multiple equilibrium branches. In the simulations we follow paths in which $\overline{\boldsymbol{C}}$ is progressively modified, starting with a field close to the identity ( $\gamma$ close to zero), and also follow paths in which $t$ changes.

While this numerical strategy is very robust, fast, and flexible, a word of caution should be mentioned: the numerically exploration of embeddability for large shape deformations must be done carefully. Embeddability may be monitored by checking numerically if $E_{m} / t=$ $(1 / t) \int_{\Gamma_{0}} w_{m} d S_{0}$ tends to zero as $t \rightarrow 0$, which requires very accurate energy minimization. The errors associated with the numerical discretization (the mesh size) and with the energy minimization algorithm must be carefully controlled for meaningful results. However, this is challenging due to the large mesh distortions associated with large pellicle shears and the ill-conditioning typical of the mechanics of very thin shells.

\subsection{Isometric embeddings beyond the singularity}

We first investigate the pseudo-sphere beyond embeddability, as reported in Figure 8, for NEPs of different thickness. We find that before the geometric singularity is reached $(\bar{\gamma}<$ 1), the theory of NEPs provides a good approximation to the isometric embedding found directly in the previous section, with very small scaled membrane energy, i.e. very small noncompliance with isometry. We observe that as $\bar{\gamma}$ increases, but remains smaller than 1 , the scaled membrane energy slightly increases. We have checked by mesh refinement that this is due to the finite element discretization errors, as more closely analyzed later. As expected, the geometric singularity manifests itself in the context of NEPs as frustrated configurations, which buckle into different symmetry-breaking shapes despite the fact that the target metric is axisymmetric. At the initial stages beyond $\bar{\gamma}=1$, the system remains axisymmetric and stores increasingly large amounts of scaled membrane energy. At a certain point, the system 


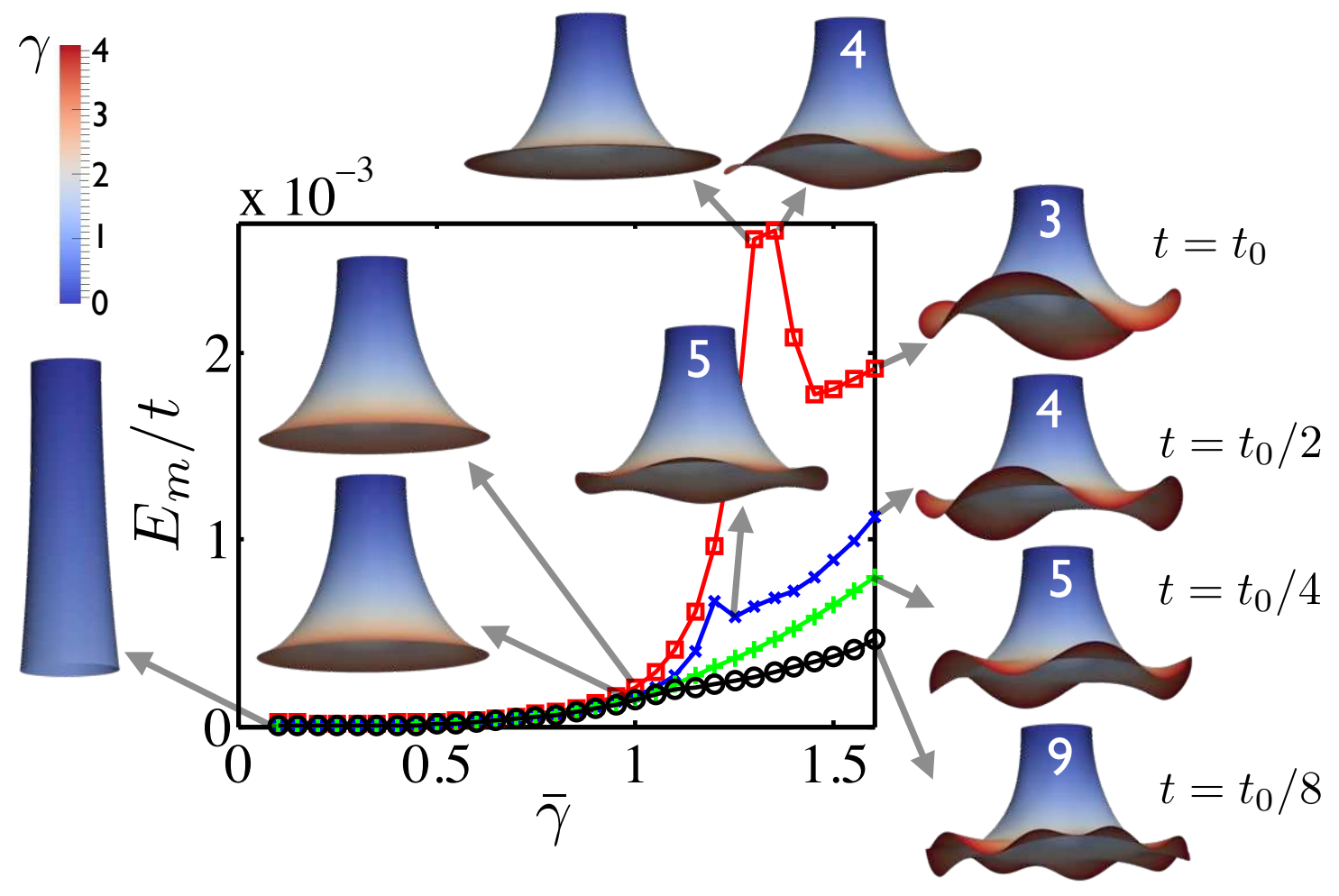

Figure 8: The pseudo-sphere beyond embeddability. We consider a pellicle shear distribution given by $\gamma=-\bar{\gamma}\left(L_{0} / R_{0}\right) \xi$ for several values of $\bar{\gamma} \geq 0$, i.e. we follow the horizontal axis in the phase diagram in Figure 6 from 0 towards negative values. At $\bar{\gamma}=1$, we hit the geometric singularity, and then further increase this parameter beyond embeddability. We examine NEPs of different thicknesses $\left(t_{0} / R_{0}=0.05\right)$, and report on the scaled membrane energy $E_{m} / t$ and the resulting morphologies. The number of downward petals is marked on the buckled configurations. 

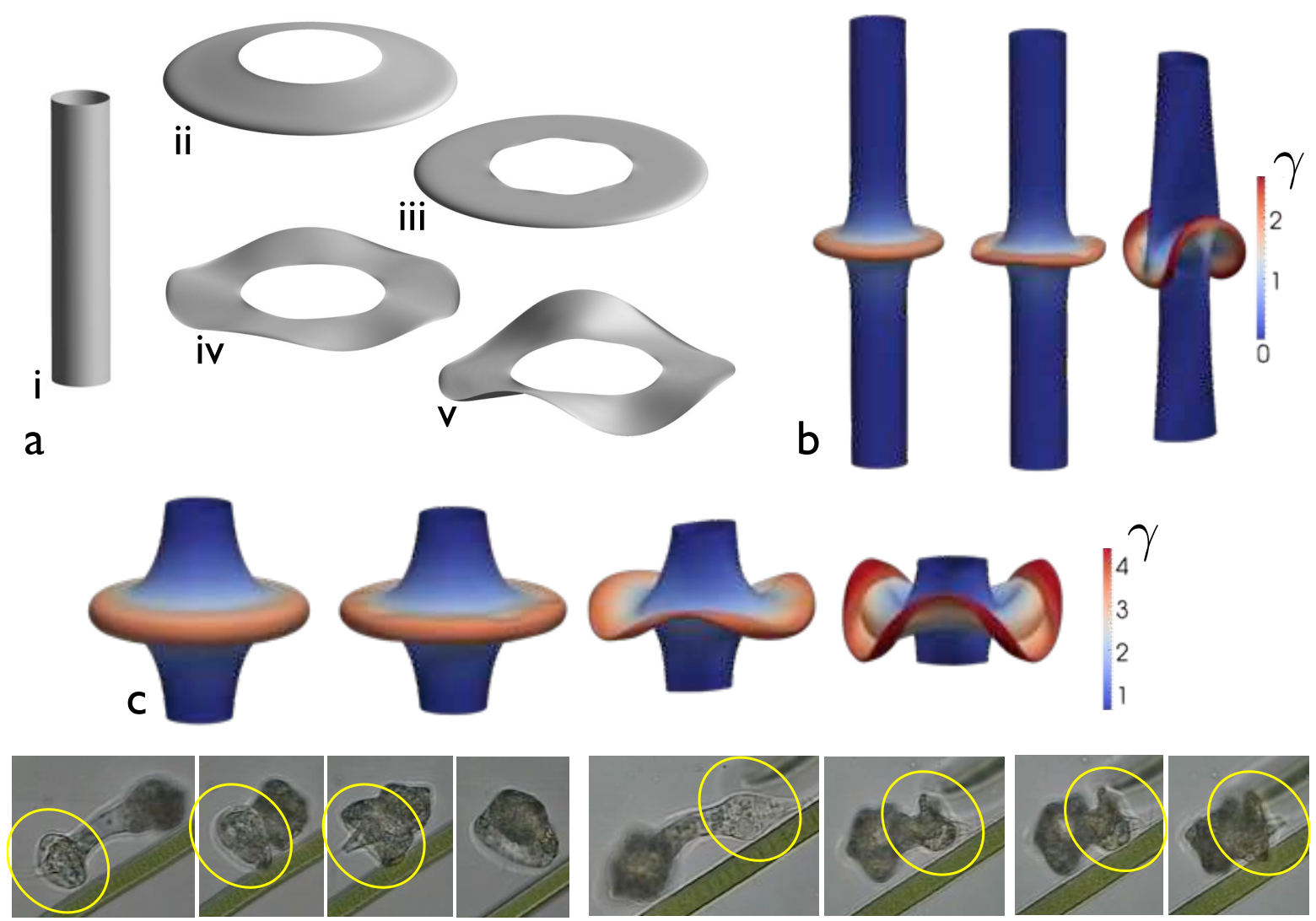

d
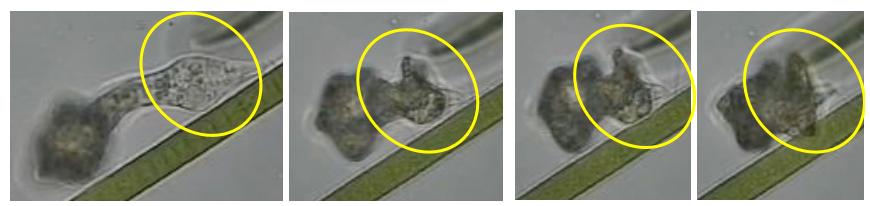

e

Figure 9: Examples of cylindrical pellicles of thickness $h / R_{0}=0.01$ brought beyond the geometric singularity (a-c). In (a), (i) is subjected to a pellicle shear following Eq. (13), which results in cones or flat annuli if exactly embedded. In (b) and (c), a Gaussian pellicle distribution as in Eq. (22) of different width and intensity is prescribed, resulting in buckled configurations with self-intersections. An anomalous euglenid, displaying abrupt shape changes and collapsed conformations is shown in (d) and (e). Original frame images courtesy of Francisco Pujante. 
partially relaxes by buckling into petaled configurations, for which deviation from isometry grows at a slower rate. While before the geometric singularity the behavior of the system is only slightly dependent on $t$, beyond the singularity the dependence is strong, in terms of the buckling point and the morphology (e.g. number of petals). We observe that thinner NEPs exhibit finer features and are able to better approximate isometry. For a fixed thickness, we also observe mode switching, by transitioning from 4 to 3, or from 5 to 4 petals. Note the very large target shear strains imposed to the system, of up to $400 \%$.

Figure 9 (a-c) shows a gallery of NEPs subject to axisymmetric pellicle shears and brought beyond the geometric singularity. Theoretically, the target metric in (a-ii) is isometrically embeddable as a flat annular disk. Instead, the finite thickness NEP stretches its outer rim, while compressing its inner rim and remaining axisymmetric. This is consistent with the embeddability condition in Eq. (15). Upon further shearing, as in the previous example, the NEP breaks symmetry and develops a wavy buckled state. A similar behavior is observed when a localized pellicle shear distribution is brought beyond the geometric limits of embeddability. A small amplitude localized wrinkling initially develops, and then switches to large-amplitude deformations that affect the whole system, and lead to self-intersecting conformations. Strikingly, these deformation modes bear similarity with those of an anomalous euglenid shown in Figure 9 (d,e). Although most euglenids exhibit smooth motions seemingly without geometric instabilities, this particular specimen undergoes abrupt shape changes, which are indicative of excessive actuation strains, buildup of stretching energy, and sudden release by buckling. The resulting conformations are collapsed, and exhibit re-entrant folds. This seems to indicate that not only geometry, but also mechanics, are important in understanding the motility of euglenids.

\subsection{Bending and twisting}

To mimic the bending and torsional motions of euglenids and after some experimentation, we examine here a strategy based on helical patterns of pellicle shear, while still considering as reference pellicle a cylindrical surface with the pellicle strips aligned with the symmetry axis of the cylinder. For this purpose, we define a helix by its azimuthal angle as a function of longitudinal coordinate, $\theta_{0}^{+}(u)=2 \pi u / B$, where $B$ is the pitch of the helix. If $B=L_{0}$, the helix performs a full turn around the cylindrical reference pellicle. We define a second

helix $\theta_{0}^{-}(u)=2 \pi u / B+\pi$, symmetric to the previous helix with respect to the axis of the cylinder. At each point of the reference pellicle labelled by $(u, v)$, its azimuthal angle can be written as $\theta(v)=v / R_{0}$. By denoting with $\Delta \theta^{ \pm}(u, v) \in[0, \pi]$ the magnitude of the angle discrepancy between $\theta(v)$ and $\theta_{0}^{ \pm}(u)$, we define

$$
\gamma(u, v)=A^{+} \exp \left[-\left(\Delta \theta^{+}(u, v) / C\right)^{2}\right]+A^{-} \exp \left[-\left(\Delta \theta^{-}(u, v) / C\right)^{2}\right],
$$

where $A^{ \pm}$characterizes the strength of shear in the \pm helices, and $C$ the lateral spread of the shear distribution around the helices. Figure 10 (a) provides an illustration for $A^{+}=0.25$, $A^{-}=-0.5, B=L_{0}$ and $C=0.3$. 

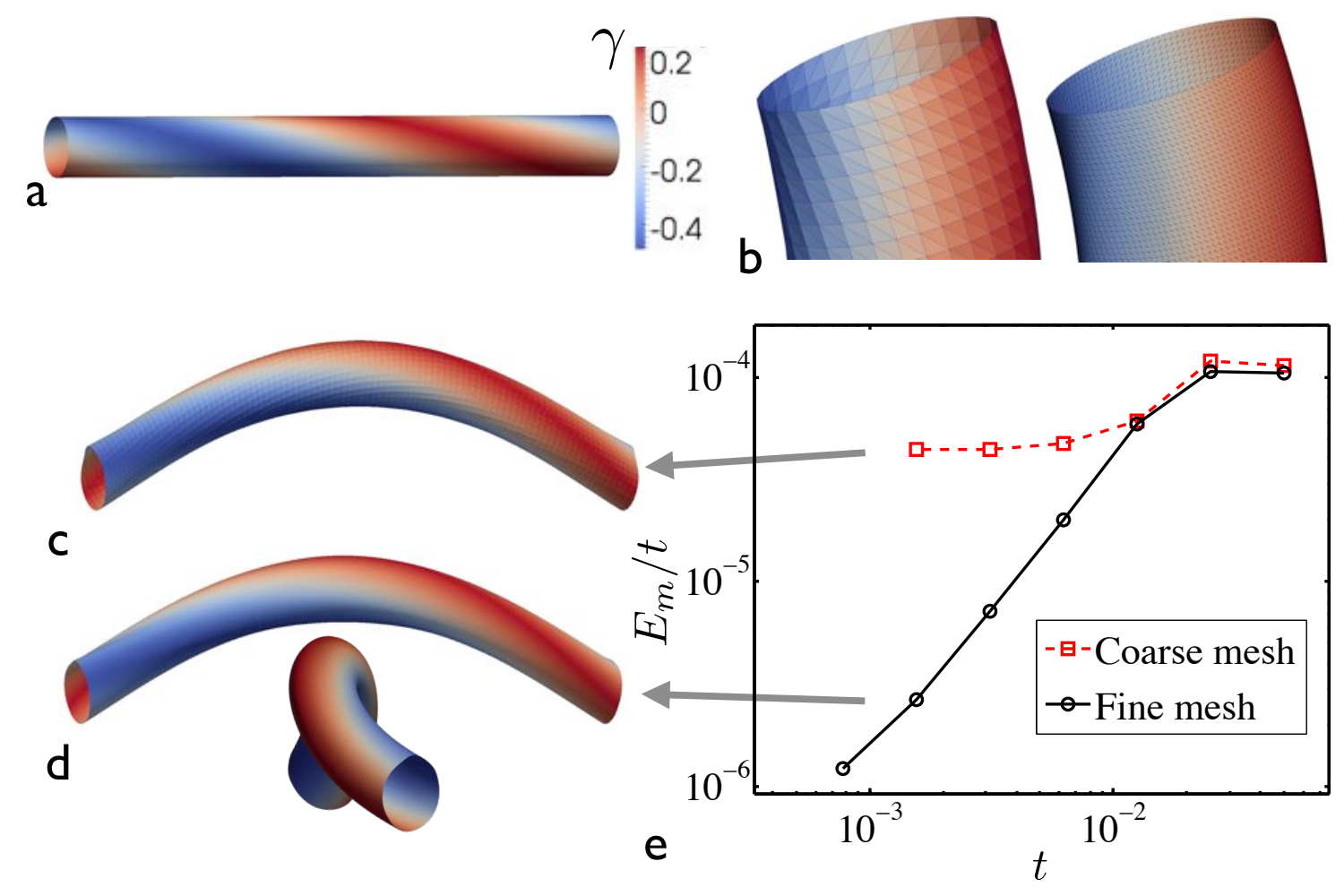

Figure 10: Numerical test of embeddability for a non-axisymmetric target metric. A reference cylindrical pellicle of unit radius is subjected to a shear distribution shown in (a), with a spiral region with lateral Gaussian modulation of positive pellicle shear (red), and an opposing spiral region with a larger negative shear (blue). We test two meshes (coarse and fine) shown in (b), and minimize the NEP energy for decreasing shell thickness $t$. The resulting deformed shapes for a given thickness and the coarse and fine meshes are shown in (c) and (d). The scaled membrane energy $E_{m} / t$ is represented against the shell thickness in a log-log scale in (e). 

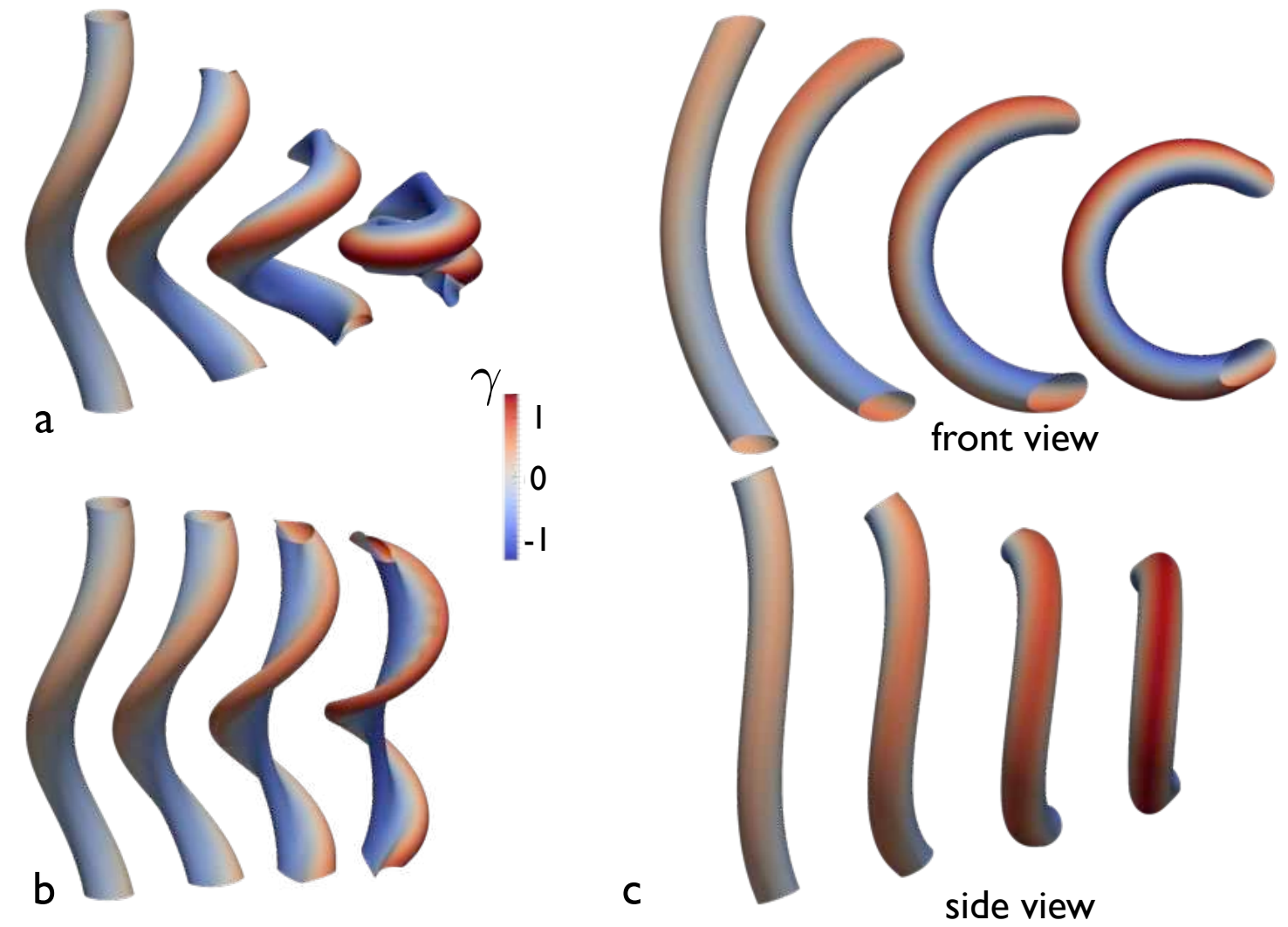

Figure 11: Deformations resulting from the target pellicle shear in Eq. (26), with parameters $A^{+}=-A^{-}=1.5, C=0.3$, and $B=L_{0}(\mathrm{a}, \mathrm{b})$ or $B=2 L_{0}(\mathrm{c})$. The NEP thickness is $t=t_{0}=0.05 R_{0}$ in (a,c) and $t=t_{0} / 4$ in (b).

In general, such a pellicle shear distribution produces a combination of bending and torsion of the cylindrical surface, as shown by the numerical results of NEP illustrated in Figure $10(\mathrm{~d})$. The immediate question that arises is whether such a non-axisymmetric target metric is embeddable, without stretch. We analyze this by numerically tracking the scaled membrane energy $E_{m} / t$ as $t \rightarrow 0$, which should converge to zero if the target metric can be exactly realized, see Figure 10 (e). We find that for a coarse mesh, the scaled membrane energy does not reduce as the shell becomes thinner. However, by refining the mesh significantly, we find that this is due to the discretization errors introduced by the finite element approximation, which can be avoided systematically by mesh refinement. The convergence of the scaled membrane energy to zero for the finer mesh provides strong numerical evidence that indeed the target metric is embeddable without membrane energy.

We now examine further the actuation mechanism embodied in Eq. (26), and consider stronger pellicle shears. Figure 11 shows results in which $A^{+}=-A^{-}$. We find that if the opposing helical patterns of shear in the cylinder are very dissimilar in magnitude, the NEP is very prone to buckling. We observe that with a short pitch, (a), the cylinder strongly bends and twists, folding onto itself at later stages. For a longer pitch, (c), one can achieve 
significant bending with little twist. If the NEP is very thin, (b), instead of bending and twisting as a whole, while keeping the cross-section close to circular, the system relaxes by severely distorting the cross section, at the expense of little global bending actuation. These results suggest that this mechanism can accomplish large planned bending and torsional motions if cross-sectional instabilities are controlled. Furthermore, other numerical tests indicate that for a given strength of the pellicle shear, $\left|A^{ \pm}\right|$, it is possible to adjust the pitch $B$ so that the resulting deformation is pure bending, without twist. While this observation is potentially interesting in applications, we note that in movies of motile euglenids bending and twisting motions of the cell body appear to be systematically coupled, suggesting the cell does not fine tune the pitch in this way.

\section{Conclusions}

We have studied the possibilities and limitations of a shape morphing mechanism for thin surfaces, inspired by the active envelope of euglenids, called pellicle. This mechanism consists of locally shearing in-plane the surface along predefined directions. Non-uniform actuation leads to curvature and shape changes, as prescribed by Gauss' Egregium theorem. Under the hypothesis of axisymmetry, and focusing on a cylindrical pellicle modeling the cell's elongated body, we have provided simple equations to find isometric embeddings, i.e. surfaces that exactly realize the prescribed shear without stretching, and characterized the embeddability restrictions. We have then examined the most elementary shapes of zero and constant Gaussian curvature, as well as localized deformation. These shapes are seen in experimental observations, as euglenids round or execute metaboly. We have found that there is a large family of continuously accessible shapes, highlighting the versatility of this actuation mechanism. Some of these canonical shapes have been also achieved by other methods to prescribe a target metric (Marder and Papanicolaou, 2006; Amar et al., 2012).

By modeling the pellicle as a non-Euclidean plate, we have examined the mechanics of the euglenoid surface morphing strategy when it is brought beyond the geometric singularity of embeddability, resulting in buckling patterns that try to accommodate the geometric frustration by developing convoluted shapes. We have related some of these configurations to the shapes adopted by an anomalous euglenid, with a strong tendency to buckling. Beyond axisymmetric actuation, we have proposed helical actuation patterns of shear, and provided numerical evidence that they can lead to exact isometric embeddings, i.e. produce non-axisymmetric deformations without stretch. We have shown that this mechanism can accomplish large amplitude bending and twisting of an elongated tubular pellicle, as seen in in vivo observations of euglenids, e.g. Figure $1 \mathrm{~B}$. It is also possible to combine the different elementary deformations examined here in different parts of a tubular pellicle to access a large repertoire of shapes, which suggests a possible concept of soft robotic arm for which the analysis of motion planning problem would be very interesting.

In the NEP model considered here, the underlying material model is isotropic, while it is clear that the pellicle is most likely mechanically anisotropic, see Figure 2. A more realistic material model, together with quantitative mechanical testing of euglenids, could provide 
further information about the mechanics of metaboly, including the force generation by molecular motors, or whether some cell shapes can be attributed to geometric instabilities. The results presented here could could help investigate the biophysical basis of behavior, e.g. the link between sensory stimuli and actuation, on a single cell minimal model system. Besides helping understand the motility of euglenids, these results may provide the background for man-made soft machines abiding by these principles. These could be made of chiral smectic C elastomers (Hiraoka et al., 2005; Adams et al., 2007), which when prepared in thin films have been shown to produce significant in-plane shear upon heating. For this purpose, it may be instructive to analyze the possibilities and limitations of different reference pellicle configurations, such as flat patches, or surfaces with a more intricate pellicle texture.

\section{Acknowledgements}

We thank Francisco Pujante for providing movies of euglenids. M.A. acknowledges the support of the European Research Council (FP7/2007- 2013)/ERC Grant Agreement nr 240487, and of the Generalitat de Catalunya though the prize "ICREA Academia" for excellence in research. This work was completed while A.D.S. was participating in the programme "The Mathematics of Liquid Crystals" as a visiting fellow of the Isaac Newton Institute for Mathematical Sciences of the University of Cambridge, whose hospitality and financial support is gratefully acknowledged.

\section{References}

Adams, J., Conti, S., DeSimone, A., 2007. Soft elasticity and microstructure in smectic C elastomers. Continuum Mechanics and Thermodynamics 18, 319-334.

Amar, M. B., Müller, M. M., Trejo, M., 2012. Petal shapes of sympetalous flowers: the interplay between growth, geometry and elasticity. New Journal of Physics 14 (8), 085014.

Armon, S., Efrati, E., Kupferman, R., Sharon, E., 2011. Geometry and mechanics in the opening of chiral seed pods. Science 333 (6050), 1726-1730.

Arroyo, M., Belytschko, T., 2002. An atomistic-based finite deformation membrane for single layer crystalline films. Journal of the Mechanics and Physics of Solids 50, 1941-1977.

Arroyo, M., Belytschko, T., 2004. Finite element methods for the non-linear mechanics of crystalline sheets and nanotubes. Int J Numer Meth Eng 59, 419-456.

Arroyo, M., Heltai, L., Millán, D., DeSimone, A., 2012. Reverge engineering the euglenoid movement. Proc Natl Acad Sci USA 109, 17874-17879. 
Ben Belgacem, H., Conti, S., DeSimone, A., Müller, S., 2000. Rigorous bounds for the fopplvon karman theory of isotropically compressed plates. Journal of Nonlinear Science 10, 661-683.

Bhattacharya, K., DeSimone, A., Hane, K., James, R., Palmstrom, C., 1999. Tents and tunnels on martensitic films. Materials Science and Engineering A 273, 685-689.

Camacho-Lopez, M., Finkelmann, H., Palffy-Muhoray, P., Shelley, M., 2004. Fast liquidcrystal elastomer swims into the dark. Nature Materials 3 (307-310).

DeSimone, A., Teresi, L., 2009. Elastic energies for nematic elastomers. European Physical Journal E 29, 191-204.

deHaan, L. T., Sánchez-Somolinos, C., Bastiaansen, C. M. W., Schenning, A. P. H. J., Broer, D. J., 2012. Engineering of complex order and the macroscopic deformation of liquid crystal polymer networks. Angewandte Chemie International Edition 51 (50), 12469-12472.

do Carmo, M., 1976. Differential geometry of curves and surfaces. Prentice-Hall.

Dobell, C., 1932. Antony van Leeuwenhoek and his "Little Animals". Dover, New York.

Efrati, E., Sharon, E., Kupferman, R., 2009. Elastic theory of unconstrained non-euclidean plates. Journal of the Mechanics and Physics of Solids 57, 762-775.

Feinberg, A. W., Feigel, A., Shevkoplyas, S. S., Sheehy, S., Whitesides, G. M., Parker, K. K., 2007. Muscular thin films for building actuators and powering devices. Science 317 (5843), $1366-1370$.

Fletcher, D. A., Theriot, J. A., 2004. An introduction to cell motility for the physical scientist. Phys Biol 1, T1-T10.

Fukunaga, A., Urayama, K., Takigawa, T., DeSimone, A., Teresi, L., 2008. Dynamics of electro-opto-mechanical effects in swollen nematic elastomers. Macromolecules 41, 93899396.

Han, Q., Hong, J.-X., 2006. Isometric Embedding of Riemannian Manifolds in Euclidean Spaces. Vol. 130. American Mathematical Society (Math. Surveys and Monographs).

Hiraoka, K., Sagano, W., Nose, T., Finkelmann, H., 2005. Biaxial shape memory effect exhibited by monodomain chiral smectic c elastomers. Macromolecules 38 (17), 7352-7357.

Hu, Z., Zhang, X., Li, Y., 1995. Synthesis and application of modulated polymer gels. Science $269,525-527$.

Jager, E. W. H., Smela, E., Inganäs, O., 2000. Microfabricating conjugated polymer actuators. Science 290 (5496), 1540-1545. 
Kim, J., Hanna, J. A., Byun, M., Santangelo, C. D., Hayward, R. C., 2012. Designing responsive buckled surfaces by halftone gel lithography. Science 335 (6073), 1201-1205.

Klein, Y., Efrati, E., Sharon, E., 2007. Shaping of elastic sheets by prescription of noneuclidean metrics. Science 315, 1116-1120.

Kwon, G. H., Park, J. Y., Kim, J. Y., Frisk, M. L., Beebe, D. J., Lee, S.-H., 2008. Biomimetic soft multifunctional miniature aquabots. Small 4 (12), 2148-2153.

Leander, B. S., 2008. Euglenida. euglenids or euglenoids. Version 11 September 2008. http://tolweb.org/Euglenida/97461/2008.09.11 in The Tree of Life Web Project, http://tolweb.org/.

URL http://tolweb.org/Euglenida/97461

Leander, B. S., Witek, R. P., Farmer, M. A., 2001. Trends in the evolution of the euglenid pellicle. Evolution 55, 2215-2235.

Lewicka, M., Reza Pakzad, M., 9 2011. Scaling laws for non-euclidean plates and the w2,2 isometric immersions of riemannian metrics. ESAIM: Control, Optimisation and Calculus of Variations 17, 1158-1173.

Liang, H., Mahadevan, L., 2009. The shape of a long leaf. Proceedings of the National Academy of Sciences 106, 22049-22054.

Marder, M., 2003. The shape of the edge of a leaf. Foundations of Physics 33, 1743-1768.

Marder, M., Papanicolaou, N., 2006. Geometry and elasticity of strips and flowers. Journal of Statistical Physics 125, 1065-1092.

Marsden, J., Hughes, T., 1983. The mathematical foundations of elasticity. Prentice-Hall.

Modes, C. D., Bhattacharya, K., Warner, M., 2011. Gaussian curvature from flat elastica sheets. Proceedings of the Royal Society A: Mathematical, Physical and Engineering Science 467 (2128), 1121-1140.

Nawroth, J. C., Lee, H., Feinberg, A. W., Ripplinger, C. M., McCain, M. L., Grosberg, A., Dabiri, J. O., Parker, K. K., 2012. A tissue-engineered jellyfish with biomimetic propulsion. Nat Biotech 30, 792-797.

Nechaev, S., Voituriez, R., 2001. On the plant leaf's boundary, 'jupe à godets' and conformal embeddings. Journal of Physics A: Mathematical and General 34 (49), 11069.

Sawa, Y., Urayama, K., Takigawa, T., DeSimone, A., Teresi, L., 2010. Thermally driven giant bending of liquid crystal elastomer films with hybrid alignment. Macromolecules 43, 4362-4369. 
Sharon, E., Efrati, E., 2010. The mechanics of non-euclidean plates. Soft Matter 6, 56935704.

Sharon, E., Marder, M., Swinney, H. L., 2004. Leaves, flowers and garbage bags: Making waves. American Scientist 92, 254-261.

Sharon, E., Roman, B., Marder, M., Shin, G.-S., Swinney, H. L., 579 2002. Mechanics: Buckling cascades in free sheets. Nature 419, 579.

Suzaki, T., Williamson, R. E., 1985. Euglenoid movement in euglena fusca: Evidence for sliding between pellicular strips. Protoplasma 124, 137-146.

Suzaki, T., Williamson, R. E., 1986. Reactivation of the euglenoid movement and flagellar beating in detergent-extracted cells of astasia longa: different mechanisms of force generation are involved. J Cell Sci 80, 75-89.

Triemer, R. E., 1999. The euglenoid project, http://euglena.msu.edu/. URL http://euglena.msu.edu/

Urayama, K., 2012. Switching shapes of nematic elastomers with various director configurations. Reactive and Functional Polymers.

Warner, M., Modes, C. D., Corbett, D., 2010. Suppression of curvature in nematic elastica. Proceedings of the Royal Society A: Mathematical, Physical and Engineering Science 466 (2124), 3561-3578.

Warner, M., Terentjev, E., 2007. Liquid crystal elastomers. Oxford University Press (International Series of Monographs on Physics 120).

White, T. J., Tabiryan, N. V., Serak, S. V., Hrozhyk, U. A., Tondiglia, V. P., Koerner, H., Vaia, R. A., Bunning, T. J., 2008. A high frequency photodriven polymer oscillator. Soft Matter 4, 1796-1798. 